\title{
Literatura médica e a ética em pesquisa: desafios para a prevenção quaternária
}

\author{
Medical literature and ethics in research: challenges for the quaternary prevention

\section{La literatura médica y la ética en pesquisa: retos para la prevención cuaternaria} \\ Hamilton Lima Wagner. Secretaria Municipal de Saúde. Curitiba, PR, Brasil. hamiltonw@uol.com.br (Autor correspondente) \\ Cynthia Ramos Soares. Secretaria Municipal de Saúde. Curitiba, PR, Brasil. cynmedi@yahoo.com.br \\ João Victor Moreira. Secretaria Municipal de Saúde. Curitiba, PR, Brasil. jvmoreira@gmail.com
}

\section{Resumo}

Este artigo discute a qualidade das evidências científicas publicadas no campo da medicina. Atualmente, existe uma dificuldade em avaliar a qualidade dessas publicações, visto que grandes ensaios clínicos são patrocinados pela indústria da saúde, em especial a indústria farmacêutica, a qual exige cláusula de confidencialidade dos dados brutos obtidos nas pesquisas. Isso gera importantes repercussões tanto para a prática dos profissionais de saúde como para a produção de diretrizes de atenção à saúde. Diante dessa incerteza de informações em saúde, a prevenção quaternária surge como uma proposta para fortalecer a relação médico-paciente como espaço para atividades preventivas e outras ações em saúde.

\section{Abstract}

This article discusses the quality of scientific evidence published in the medical field. Currently, there is a difficulty in assessing the quality of these publications, since large clinical trials are sponsored by the health industry, particularly the pharmaceutical industry, which requires confidentiality clause of the raw data obtained in the research. This creates significant implications for both the practice of health professionals and the production of health care guidelines. Given these uncertainties of health information, quaternary prevention emerges as a proposal to strengthen doctor-patient relationship as space for preventive activities and other health actions.

\section{Resumen}

En este artículo se discute la calidad de la evidencia científica publicada en el campo de la medicina. En la actualidad, hay una dificultad en la evaluación de la calidad de estas publicaciones, ya que los grandes ensayos clínicos son patrocinados por la industria de la salud, en particular la industria farmacéutica, que requiere cláusula de confidencialidad de los datos brutos obtenidos en la investigación. Esto crea importantes implicaciones tanto para la práctica de los profesionales de la salud y como para la producción de guías de atención de salud. Dada esta incertidumbre de la información en la salud, la prevención cuaternaria surge como una propuesta para fortalecer la relación médico-paciente como espacio para actividades preventivas y otras acciones de salud.

Palavras-chave: Prevenção Quaternária Pesquisa Biomédica Ética Conflito de Interesses

\section{Keywords:} Quaternary Prevention Biomedical Research Ethics Conflict of Interes

\section{Palabras clave:} Investigación Biomédica Ética

Conflicto de Intereses

Fonte de financiamento:
declaram não haver.
Parecer CEP:
não se aplica.
Conflito de interesses:
declaram não haver.
Procedência e revisão por pares:
revisado por pares.
Recebido em: 25/09/2014.
Aprovado em: 13/04/2015.


A qualidade da literatura científica tem estado na pauta das discussóes no campo da medicina, havendo questionamentos sobre a qualidade e segurança dos textos. Em 1993, quando Sackett et al. ${ }^{1}$ lançam os fundamentos da Medicina Baseada em Evidências (MBE) aparentemente estavam fornecendo o caminho para a definiçáo de como deveria ser produzida uma informação de qualidade.

No entanto, os instrumentos desenhados para melhorar a qualidade das pesquisas foram apropriados pela indústria da saúde, particularmente pela indústria farmacêutica, sendo que muitas das informaçôes disponíveis atualmente nas várias publicaçóes utilizam inadequadamente os conceitos inicialmente propostos. ${ }^{2,3}$ Isso tem repercussóes para as diretrizes fundamentadas em estudos que podem ser questionados, seja pela ocultação de dados, seja pela adoção de orientações e metas que desconsideram o bem estar dos pacientes. ${ }^{4-6}$

Há anos os vieses de interesse da indústria farmacêutica vêm impactando a literatura médica e desde 1950 existem documentos mostrando distorçóes graves na conduta da indústria da saúde, na busca do lucro a qualquer custo. Um exemplo clássico é o escândalo da talidomida, droga desenvolvida na Alemanha, cujos trabalhos científicos que mostravam as malformaçóes decorrentes de seu uso foram ignorados na época pelo seu fabricante. Este negou o problema e criou empecilhos na justiça durante anos para que, finalmente, fossem reconhecidos os danos causados pela droga indicada para tratamento de enjoos na gestação.

Outro importante exemplo a ser mencionado é a metanálise das pesquisas feitas pelos fabricantes dos medicamentos risperidona, olanzapina e quetiapina ${ }^{8}$ no qual as três companhias indicavam em ensaios clínicos que suas drogas eram melhores que as demais. Tal fato sugere que boa parte dos estudos publicados apresentam problemas metodológicos, porém isso náo impede o marketing extensivo sobre a eficiência desses medicamentos e a comparaçáo benéfica que a pesquisa do laboratório demonstrou.

Conhecer o desenho e o objetivo de uma pesquisa facilita ao leitor o entendimento dos resultados esperados. Esta informação, contudo, é geralmente ocultada, permitindo que os autores trabalhem e manipulem os dados de acordo com os seus interesses e descrevam resultados inesperados como sendo o foco da pesquisa. ${ }^{3}$ Tal situação conduz a profundas distorçóes e talvez explique por que muitas vezes os profissionais de saúde se deparam na prática com resultados inesperados e efeitos adversos não previstos. ${ }^{9}$

Uma pesquisa bem feita deve ter um desenho tal que permita ao pesquisador provar que a sua teoria está errada, pois o objetivo real da pesquisa científica é afastar a hipótese. Em outras palavras, deve levar à busca das imperfeiçóes e não de resultados favoráveis, pois ao se procurar apenas a confirmação da hipótese existe a probabilidade de, involuntariamente, se distorcer os dados. ${ }^{?}$

Ioannidis ${ }^{10}$ e Senn ${ }^{11}$ demonstram em seus artigos como a estatística pode ser utilizada para confirmar resultados que, quando analisados de forma adequada, não seriam verdadeiros. Além disto, expressar o nível de significância de uma pesquisa como probabilidade de erro menor que $5 \%$ (ou p $<0,05$ ) é de pouco auxílio para a tomada de decisão compartilhada.

Um conceito central, que muitas vezes leva o estudante da área da saúde a confundir-se neste emaranhado de fórmulas e indicaçóes, é a regressão à média. Este se fundamenta no princípio da homeostase que caracteriza todos os sistemas vivos que tendem a se equilibrar e a trabalhar ativamente para que isto aconteça; em outras palavras, voltar à média. As pesquisas que não consideram o princípio da regressão à média têm dentro de seu desenho um erro inato que tira muito de sua credibilidade, gerando resultados que podem ser fruto do trabalho interno do organismo, ${ }^{10}$ que impedem uma compreensão apropriada dos efeitos do protocolo em estudo.

A presença de pessoas ligadas à indústria e à atividades políticas, tanto nos comitês de pesquisa como nas coordenaçôes de proposiçâo de diretrizes, gera uma combinação de informaçôes problemáticas, dificultando aos profissionais vinculados diretamente a assistência à saúde uma tomada de decisão adequada. ${ }^{3}$ Parte da responsabilidade acaba recaindo sobre os profissionais de saúde, que por falta de uma maior apropriação de epidemiologia, dos desenhos de pesquisa ou de como os estudos são publicados, acabam aceitando informaçôes com muitos vieses, o que pode expor pacientes a um risco elevado. Por exemplo, pesquisas informam que a medicina é a terceira causa de morte nos Estados Unidos, onde o rigor estatístico é melhor, perdendo apenas para as doenças cardiovasculares e as neoplasias. ${ }^{3}$

Por outro lado, a indústria da saúde não se concentra apenas na indicaçáo de medicamentos, mas também interfere na definiçẫo de critérios de saúde. ${ }^{11,12}$ Muitas pesquisas são realizadas para modificar parâmetros de normalidade, sem que haja significativa melhora da qualidade/quantidade de vida das pessoas. ${ }^{13}$ St-Onge ${ }^{14}$ questiona em seu livro se "Estamos 
todos loucos?", pois tal é a quantidade de novos diagnósticos surgidos em psiquiatria e o percentual de pessoas em uso de medicamentos para a saúde mental no Canadá e nos Estados Unidos.

Cada vez mais são almejados marcadores de saúde "rigorosos", sem a preocupação com o impacto na qualidade de vida das pessoas. Por exemplo, nos anos 1970 o critério para o diagnóstico de diabetes mellitus era duas glicemias de jejum acima de $140 \mathrm{mg} / \mathrm{dl}$; nos anos 1990 este número foi reduzido para duas glicemias acima de $126 \mathrm{mg} / \mathrm{dl}$; e atualmente há grandes defensores de que a normalidade é abaixo de $100 \mathrm{mg} / \mathrm{dl} .{ }^{15,16}$ Ainda em relação à diabetes, existe a tendência de se tratá-la pelos níveis de hemoglobina glicada, havendo a recomendação de alcançar valores abaixo de $7 \mathrm{mg} / \mathrm{dl}$ para todos os pacientes diabéticos, sendo considerados como desejáveis valores próximos de $6 \mathrm{mg} / \mathrm{dl} .{ }^{17}$ Recente artigo publicado no JAMA ${ }^{18}$ mostrou que para pacientes com diabetes tipo 2 acima de 60 anos há um ganho apenas limítrofe para os que buscam estes níveis, em comparação com os que tem uma hemoglobina glicada abaixo de $9 \mathrm{mg} / \mathrm{dl}$ em uso de metformina. Tal informaçáo implica em uma mudança drástica na qualidade de vida destas pessoas, em um uso menor de medicamentos e em uma relação mais tranquila entre a equipe de saúde e os pacientes diabéticos. $\mathrm{O}$ exemplo da diabetes discutido acima traz reflexôes sobre os pontos de corte que definem doenças, até onde se deve tratar ou náo as pessoas (metas), assim como, o impacto das intervençóes sobre a qualidade de vida e a autonomia das pessoas.

Em verdade, muitas situaçôes de vida normais passaram a ser tratadas como patologias (i.e. a gestação, a puericultura, a menopausa, a osteoporose, entre outros) gerando grandes gastos e investimentos em diagnóstico e tratamentos. Estas deveriam ser consideradas dentro de um estilo de vida saudável e abordadas em orientaçôes, tanto individuais como coletivas, sobre como lidar com as diferentes fases da vida, e não serem tratadas como doença. ${ }^{10,13}$ Ainda mais relevante é a transformação de fatores de risco em patologias e o tratamento desses fatores de risco como doenças, situação que tende a produzir ansiedade e perda da qualidade de vida. ${ }^{13}$

Conceitos como sobrediagnóstico, sobretratamento e invenção de diagnósticos devem entrar na pauta de discussão dos pesquisadores médicos, da comunidade científica e dos profissionais de saúde para que se possa fortalecer a prática de uma medicina mais ética e centrada nas pessoas. ${ }^{2}$ Nesse sentido, congressos internacionais tem sido organizados com o intuito de minimizar os abusos na literatura e na pesquisa médica, sugerindo a necessidade de se repensar o modo de cuidado aos pacientes.

Em 1986, Jamoulle ${ }^{19}$ apontava a necessidade de se evitar os excessos da medicina e oferecer uma melhor atenção à saúde aos pacientes e comunidades, que definiu como prevenção quaternária (P4). Este tema se expandiu e atualmente ocupa espaço nas atividades da medicina de família e comunidade (MFC). ${ }^{20}$ Deste debate surgiu a necessidade de mudança de paradigma, de incorporar na atenção à saúde não apenas as evidências científicas, mas também a decisão compartilhada.

Para tanto, é necessário que o desenho das pesquisas científicas seja disponibilizado para uma avaliação adequada e independente. Isso permitiria que os dados brutos das pesquisas pudessem ser analisados, o que facilitaria o cálculo dos números necessários de pacientes a tratar (NNT) e dos números necessários de pacientes sob risco de sofrer danos decorrentes do tratamento (NND). Esse contexto de maior transparência com os dados das pesquisas facilitaria uma honesta e tranquila decisão compartilhada com os pacientes, por meio de uma linguagem mais clara e compreensível.

\section{Referências}

1. Sackett DL, Straus SE, Richardson WS, Rosenberg W, Haynes RB. Evidence-based medicine. New York: Churchill Livingstone; 1997.

2. Moynihan R, Doran E, Henry D. Disease mongering is now part of the global health debate. PLoS Med. 2008;5(5):e106. http://dx.doi.org/10.1371/journal.pmed.0050106. PMid:18507498.

3. Gøtzsche PC. Deadly medicines and organised crime: how big pharma has corrupted healthcare. Cornwall: Radcliffe Publishing; 2013.

4. Every-Palmer S, Howick J. How evidence-based medicine is failing due to biased trial and selective publication. J Eval Clin Pract. 2014; 20:908-914.

5. Greenhalgh T, Howick J, Maskrey N. Evidence based medicine: a movement in crisis? BMJ. 2014; 348:g3725. http://dx.doi.org/10.1136/bmj.g3725. PMid:24927763.

6. Akst J. Dealing with irreproducibility. The Scientist: Exploring Life, Inspiring Innovation [Internet]. 2014. [acesso em 2015 Oct 18] Disponível em: http://www.the-scientist.com/?articles.view/articleNo/39654/title/Dealing-with-Irreproducibility/

7. Brynner R, Stephens T. Dark remedy: the impact of thalidomide and its revival as a vital medicine. New York: Perseus Publishing; 2001. 
8. Heres S, Davis J, Maino K, Jetzinger E, Kissling W, Leucht S. Why olanzapine beats risperidone, risperidone beats quetiapine, and quetiapine beats olanzapine: an exploratory analysis of head-to-head comparison studies of second-generation antipsychotics. Amer $\mathrm{J}$ of Psychiatry. 2006(163):185-194.

9. Fletcher RH, Fletcher S. Epidemiologia clínica. Porto Alegre: Artmed; 2006.

10. Ioannidis JPA. Why most published research findings are false. PLoS Med. 2005;2(8):e124. http://dx.doi.org/10.1371/journal.pmed.0020124.

11. Senn S. Three things that every medical writer should know about statistics. The Write Stuff. 2009; 18(3):159-162. [acesso em 2015 June 18] Disponível em: http://eprints.gla.ac.uk/8107/1/id8107.pdf

12. Dear JW, Webb DJ. Disease mongering -- a challenge for everyone involved in healthcare. Br J Clin Pharmacol. 2007;64(2):122-124. http://dx.doi.org/10.1111/j.1365-2125.2006.02830.x. PMid:17214830.

13. Gérvas J, Pérez-Fernández MP. Sano y Salvo y libre de intervenciones médicas innecesarias. Barcelona; Los Libros del Lince; 2013.

14. St-Onge JC. Tous fous? Montreal: Écosociété; 2013.

15. Goroll AH, May LA, Mulley AG, editores. Prymare care medicine. Philadelphia: JB Lippincott; 1995.

16. Weiss BD, editor. 20 commom problems in primary care. San Antonio: McGraw-Hill; 1999. p. 79-120.

17. Oliveira JEP, Vencio S, editors Diretrizes da sociedade brasileira de diabetes. São Paulo: AC Farmacêutica; 2015.116 p.

18. Vijan S, Sussman JB, Yudkin JS, Hayward RA. Effect of patients' risks and preferences on health gains with plasma glucose level lowering in type 2 diabetes mellitus. JAMA Intern Med. 2014;174(8):1227-1234. http://dx.doi.org/10.1001/jamainternmed.2014.2894. PMid:24979148.

19. Jamoulle M, Berstein J, Pizzanelli Baez M, Silva A, Wagner H. Prévention quaternaire: un concept fondé sur le temps et la relation. Deuxième partie. L'étonnante diffusion internationale du concept. Médecine. 2014(10)2:75-77. http://dx.doi.org/10.1684/med.2014.1066.

20. Jamoulle M. Quaternary prevention, an answer of family doctors to overmedicalization. Intern J Health Policy Manag. 2015;4(2).61-64. http://dx.doi.org/10.15171/ijhpm.2015.24. 